\title{
A novel approach for predicting the spatial patterns of urban expansion by combining the chi-squared automatic integration detection decision tree, Markov chain and cellular automata models in GIS
}

\begin{abstract}
Urban development is a continuous and dynamic spatio-temporal phenomenon associated with economic developments and growing populations. To understand urban expansion, it is important to establish models that can simulate urbanization process and its deriving factors behaviours, monitor deriving forces interactions and predict spatio-temporally probable future urban growth patterns explicitly. In this research, therefore, we presented a hybrid model that integrates the chi-squared automatic integration detection decision tree (CHAIDDT), Markov chain (MC) and cellular automata (CA) models to analyse, simulate and predict future urban expansions in Tripoli, Libya in 2020 and 2025. First, CHAID-DT model was applied to investigate the contributions of urban factors to the expansion process, to explore their interactions and to provide future urban probability map; second, MC model was employed to estimate the future demand of urban land; third, CA model was used to allocate estimated urban land quantity on the probability map to present future projected land use map. Three satellite images of the study area were obtained from the periods of 1984, 2002 and 2010 to extract land use maps and urban expansion data. We validated the model with two methods, namely, receiver operating characteristic and the kappa statistic index of agreement. Results confirmed that the proposed hybrid model could be employed in urban expansion modelling. The applied hybrid model overcame the individual shortcomings of each model and explicitly described urban expansion dynamics, as well as the spatiotemporal patterns involved.
\end{abstract}

Keyword: Urban expansion; CHAID-DT; GIS; Remote sensing; Tripoli 